\title{
A UTILIZAÇÃO DO GRUPO DE FOCO PARA A AVALIAÇÃo dA OPINIÃO dOS CLIENTES: A EX- PERIÊNCIA DO SERVIÇO DE BIBLIOTECA E DOCUMENTAÇÃO DA ECAUSP
}

\author{
Bárbara Júlia Leitão \\ Waldomiro Vergueiro
}

\begin{abstract}
Resumo
Discute a técnica de entrevista por grupo de foco e sua utilização em ambientes de serviço de informação, partindo da literatura internacional sobre o tema. Descreve e analisa experiência, realizada na biblioteca da Escola de Comunicações e Artes da Universidade de São Paulo, utilizando essa técnica de pesquisa qualitativa. Procura demonstrar sua viabilidade para bibliotecas acadêmicas de países em desenvolvimento, defendendo que ela propicia a obtenção de resultados valiosos que podem ser utilizados para a melhoria da qualidade dos serviços prestados. Propõe a integração da técnica aos processos de planejamento bibliotecário.
\end{abstract}

Palavras-chave

Bibliotecas - Planejamento - Grupo de foco; Usuários - Entrevista - Grupo de foco; Grupo de foco.

\section{INTRODUÇÃO}

Novas instalações físicas normalmente representam um investimento substancial para bibliotecas em todo o mundo. Esse tipo de investimento é mais difícil em bibliotecas de países em desenvolvimento, nos quais a disponibilidade de recursos financeiros é sempre obtida de forma incerta. Nesses países, quando uma biblioteca consegue um novo prédio, é importante avaliar seriamente como ele será visto e otimizado pelos usuários. Freqüentemente, a decisão de construir um novo prédio é tomada sem prestar muita atenção sobre como o cliente irá encarar o novo ambiente e mesmo se este espaço estará trazendo uma vantagem real em sua vida. Os adminis- 
tradores de bibliotecas devem estar atentos à opinião dos usuários sobre o local e levá-la em consideração no planejamento dos serviços.

No entanto, é sempre difícil avaliar as opiniões dos clientes devido à falta de recursos humanos para realizar pesquisa mais complexa. Por essa razão, é importante encontrar técnicas menos dispendiosas para ouvir os clientes. Este trabalho descreve o uso de uma dessas técnicas - a técnica de entrevista por grupo de foco - aplicando-a no contexto de uma biblioteca acadêmica brasileira, concluindo que ela é adequada para esse propósito.

\section{A ESCOLA DE COMUNICAÇÕES E AR- TES DA UNIVERSIDADE DE SÃO PAULO}

A biblioteca da Escola de Comunicações e Artes da Universidade de São Paulo (ECA/USP), atualmente denominada Serviço de Biblioteca e Documentação (SBD/ECA), foi fundada em 1970 e visa apoiar o ensino e a pesquisa realizados nos nove departamentos da escola (Ciência da Informação; Comunicações e Artes; Cinema, Televisão e Rádio; Jornalismo e Editoração; Artes Cênicas; Artes Plásticas; Música; Relações Públicas, Turismo e Propaganda) e a Escola de Arte Dramática, uma escola de segundo grau também mantida pela ECA/USP.

O acervo do SBD/ECA é composto por livros, teses, periódicos, partituras, discos, fitas de áudio e vídeo, filmes, fotografias, CDs, CDROMs, catálogos de exposições, peças de teatro e revistas de histórias em quadrinhos, em um total de 133.100 itens. Sua comunidade é composta pelos estudantes, professores, pesquisadores e funcionários, totalizando 2.336 pessoas. Ele atende uma média de 550 usuários por dia, tanto da Universidade de São Paulo como de outras instituições.

Em 1997, o SBD/ECA recebeu recursos financeiros da Fundação de Amparo à Pesquisa do Estado de São Paulo (FAPESP) para remodelar suas instalações, que não tinham sofrido reformas substanciais desde 1982. Esta reforma envolveu a reconstrução do espaço físico e a aquisição de novo mobiliário e computadores. Com o novo espaço, a biblioteca dispõe agora de 14 terminais com acesso à Internet, sala para estudo em grupo, cabines individuais para estudo e audição de música e visualização de vídeos, além de salas de vídeo e áudio para uso em grupo.

Uma quantia considerável foi gasta na construção do novo espaço, incluindo reconstrução, equipamentos e mobiliário: $\mathrm{R} \$$ 770.330,00 (aproximadamente 640 mil dólares).

\section{A TÉCNICA DE ENTREVISTA POR GRUPO DE FOCO}

O interesse por essa técnica de entrevista iniciou-se de maneira casual. A partir de uma conversa informal com uma pessoa que já havia trabalhado com este tipo de metodologia em agências de publicidade, sentiu-se despertar a curiosidade para a aplicação desse método em ambiente de serviços de informação. Assim, tendo em vista esse objetivo, buscou-se, na literatura especializada, trabalhos voltados para bibliotecas, especialmente acadêmicas. Isso permitiu a identificação de vários textos que tratam dessa Inf.Inf., Londrina, v. 4, n. 2, p. 95-104, jul./dez. 1999 
questão.

Na obra Service Quality in Academic Libraries, Hernon \& Altman (1996) desenvolvem todo o seu trabalho a partir das informações obtidas por meio de grupo de foco. Outra utilização da técnica pode ser encontrada em artigo de Radford (1998), no qual a autora trabalha problemas de linguagem não-verbal (corporal) entre usuários e bibliotecários de referência utilizando esta técnica de entrevista.

Rose, Stoklosa, Gray (1998) também utilizam esta técnica para analisar o stress no serviço de referência da Health Sciences Library da State University of New York. Widdows, Hensler, Wyncott (1991), por sua vez, utilizam-na para medir a opinião do usuário estudante em relação à qualidade do serviço em uma biblioteca acadêmica.

Embora o levantamento da literatura permitisse a identificação de poucos textos, todos tinham em comum a apresentação da técnica de entrevista por grupo de foco de maneira prática e acessível, o que colaborou para que se concluísse pela sua viabilidade na realidade das bibliotecas brasileiras. Para isso, buscou-se o embasamento teórico para a utilização correta desse tipo de pesquisa e procurou-se seguir as orientações recomendadas na bibliografia utilizada, embora adequando-as às limitações e objetivos do estudo específico.

\section{1 Descrição da metodologia}

Essa técnica de entrevista tem sua origem na década de 40, nas Ciências Sociais e nas pes- quisas de marketing. Foi utilizada pela primeira vez na ciência da informação nos anos 80 , para avaliar as atitudes dos usuários em relação aos catálogos on-line. Drabenstott (1992) define-a como um método efetivo para identificar sentimentos e convicções sobre situações, produtos e serviços, bem como a forma como esses sentimentos e convicções moldam o comportamento das pessoas. Para ele, são necessários quatro fatores para que as pesquisas sejam produtivas:

- Não deve haver indução;

- Necessitam ser específicas;

- Devem ter alcance dentro da proposta de discussão; e

- Devem ter profundidade e contexto pessoal.

Segundo Patton (1990), grupo de foco é uma entrevista formada por um pequeno grupo de pessoas com um tema específico. Os grupos são normalmente constituídos por um total de seis a oito membros, que participam de um encontro de uma e meia a duas horas de duração. $\mathrm{O}$ autor afirma que a função do grupo de foco não é resolver problemas, tomar decisões e tampouco se caracteriza como uma forma de discussão. A função primordial do grupo de foco é a entrevista em si.

Morgan (1988) defende que o grupo de foco é o uso explícito da interação para produzir dados e insights. Segundo o autor, podem-se encontrar pontos fortes e fracos nessas pesquisas:

- Pontos fortes: os grupos são fáceis de conduzir; a técnica não exige muitos recursos financeiros; mesmo que o pesquisador seja novato poderá obter muitas informações, pois 
o grupo permite que se explore tópicos e hipóteses gerais, possibilitando interação com os tópicos de interesse do pesquisador,

- Pontos fracos: não é baseado em colocações naturais; o grupo de foco não atinge o potencial dos grupos de pesquisas individuais, e certas posições de alguns indivíduos podem distorcer o estudo.

O ponto principal do grupo de foco é poder estar centralizado em um tópico preestabelecido e possuir um moderador presente. É importante lembrar que esta técnica de entrevista não produz resultados para projeções estatísticas, podendo ser utilizados tanto isoladamente como em conjunto com outros métodos.

\section{A TÉCNICA DE ENTREVISTA POR GRUPO DE FOCO NO SBD/ECA}

\subsection{Objetivos de estudo}

O objetivo deste trabalho foi analisar como o usuário estava percebendo a biblioteca no período pós-reforma. Não se tratava, assim, de realizar uma pesquisa quantitativa, mas conhecer melhor a opinião dos usuários. A discussão por grupo de foco como uma das opções existentes no âmbito das pesquisas qualitativas surgiu em função desse objetivo, parecendo constituir-se um promissor instrumento para a coleta de dados. Outro fator favorável a essa decisão foi o baixo custo dessa técnica. Nesse sentido, Morgan (1988) assevera que os próprios pesquisadores podem conduzir os grupos para que os custos sejam reduzidos.
A definição do grupo estruturou-se de forma a incluir apenas os alunos de pós-graduação que conheceram a biblioteca antes da reforma e continuaram a utilizá-la após esse período. Esta opção baseou-se na expectativa de uso maior da biblioteca por alunos de pós-graduação e em seu maior nível de exigência em relação aos serviços recebidos. Foram convidados 7 alunos de pósgraduação.

A literatura especializada justifica o número adotado, determinando um mínimo de 5 (cinco) e um máximo de 12 (doze) pessoas para a constituição do grupo (Morgan, 1988). Da mesma forma, teve-se em mente que a estrutura do grupo deve ser homogênea, os membros partilhando o mesmo nível socioeconômico e educacional. Neste aspecto, é importante salientar que a formação do grupo não se mostrou tão fácil como se esperava, devido a dificuldades de agendamento conveniente para todos os entrevistados. No entanto, como foi relatado por Widdows, Hensler, Wyncott (1991), tudo parece indicar que as dificuldades encontradas na Escola de Comunicações e Artes são comuns àquelas de outros ambientes acadêmicos.

Morgan (1988) salienta a necessidade de a discussão ser dirigida por um moderador e acompanhada por um observador, descritos da seguinte forma:

- Moderador: responsável pela condução da entrevista, não podendo interferir nas participações do grupo; deve saber o momento de reconduzir ao assunto, quando o grupo dispersa sua atenção; deve estar atento para que nenhum dos participantes lidere o gruInf.Inf., Londrina, v. 4, n. 2, p. 95-104, jul./dez. 1999 
po, incentivando os mais tímidos a emitir suas opiniões.

- Observador: responsável pelas anotações das opiniões de todos os participantes; deve ser uma pessoa com excelente capacidade de audição e concentração, além de ter capacidade para tomar notas com precisão e acuidade.

Segundo a literatura, é aconselhável que tanto o moderador como o observador sejam estranhos aos membros do grupo (Morgan, 1988). No entanto, na medida em que contar com alguma pessoa com experiência nesse tipo de entrevista significava um custo maior para o trabalho, podendo resultar até mesmo em sua inviabilidade, optou-se por ter a diretora da biblioteca no papel de mediadora. Nada leva a crer que essa decisão tenha interferido nos resultados obtidos.

O espaço escolhido para a discussão foi uma sala no recinto da própria biblioteca, adequada aos objetivos do trabalho, garantindo a disposição de todos, sem qualquer tipo de diferenciação entre os envolvidos. Nesse sentido, procurou-se seguir orientações sobre o espaço físico sugeridas por Fraser, Restrepo-Estrada (1998).

\section{2 A Entrevista}

Dos sete convidados, apenas quatro compareceram. Mesmo assim, a entrevista foi realizada, tanto por respeito aos membros presentes como por se entender que seria muito difícil marcar uma nova data. Dessa forma, teve-se a parti- cipação de 2 alunos de Música, 1 de Turismo e 1 de Ciência da Informação.

Como é praxe nesse tipo de entrevista, solicitou-se a aquiescência do grupo para realizarse a sua gravação em áudio, para o que não houve qualquer restrição. Essa opção considerou também os baixos custos envolvidos na utilização desse recurso de gravação.

Utilizou-se o seguinte roteiro de perguntas:

- Condições físicas: como o ambiente físico era visto antes da reforma e como está sendo visto hoje.

- Atendimento: se melhorou, se continua igual ou se piorou após a reforma.

- Localização do material nas estantes: se está mais fácil localizar o material.

- Serviços prestados: se os participantes sabem quais os serviços que a biblioteca oferece

- Quais são as expectativas e/ou necessidades

Apesar do roteiro, a discussão correu de forma natural, e os membros ativeram-se a uma seqüência em que um tema conduzia a outro. Somente em alguns momentos o moderador sentiu necessidade de interferir para que não houvesse dispersão do assunto.

Notou-se uma certa inibição no inicio dos trabalhos e que um dos membros do grupo exercia uma forte liderança. Porém, com o desenro- 
lar da entrevista e o posicionamento do moderador, os demais membros puderam participar da discussão sem maiores problemas. De uma certa forma, isto já era esperado, pois a literatura consultada, em geral, indica um período de abertura e acomodação de 10 a 15 minutos para que os participantes possam se conhecer e se sentir mais a vontade.

A entrevista durou 1 hora e 30 minutos, o que está de acordo com o recomendado, ou seja, de 60 a 90 minutos.

\section{PRINCIPAIS PONTOS ENFATIZADOS NA ENTREVISTA}

Demonstramos, abaixo, as opiniões dos participantes do grupo, de acordo com o roteiro determinado para que o foco da entrevista não fosse perdido.

\section{a) a biblioteca antes da reforma:}

Os participantes consideravam a biblioteca, literalmente, um "horror", pois a luminosidade não era adequada e o espaço entre as estantes era muito estreito, o que dificultava a localização dos materiais. Além de as mesas e cadeiras não serem suficientes, também não havia espaço para estudo em grupo e ao mesmo tempo não existia privacidade para o estudo individual.

A audição de discos de vinil ou a visualizacao de vídeos eram prejudicados devido ao fato de o espaco disponível para essas atividades ser compartilhado; isso sempre acabava incomodando os usuários.
Quanto à limpeza do ambiente, os participantes afirmaram que, apesar dos esforços para manutenção, a imagem era a de uma biblioteca não muito limpa.

\section{b) a biblioteca após a reforma:}

Segundo os participantes do grupo, o ambiente tornou-se bem mais agradável, iluminado, e existe prazer em ali se permanecer. Além disso, os participantes disseram que agora as pessoas estão mais cuidadosas na utilização dos materiais.

\section{c) a comunicação visual}

Os participantes a acharam muito satisfatória, pois possibilita economizar tempo para a localização dos materiais. No entanto, na opinião dos participantes, parece faltar uma indicação externa na entrada do prédio, informando a localização da biblioteca. Em relação à comunicação interna, o único ponto negativo apontado foi a falta de uma indicação para a coleção retrospectiva dos jornais.

\section{d) atendimento}

Os participantes sentem a equipe de atendimento mais alegre, solícita, trabalhando com bom humor e coesos. Isso colabora para que sejam mais gentis e transmitam simpatia para os usuários. Dessa forma, foi opinião unânime do grupo que o nível de atendimento melhorou significativamente.

Os participantes fizeram a comparação com a biblioteca antes da reforma, afirmando que o Inf.Inf., Londrina, v. 4, n. 2, p. 95-104, jul./dez. 1999 
ambiente físico anterior era inadequado e não havia condições satisfatórias de trabalho, passando uma imagem de desorganização e desarmonia entre os funcionários.

\section{e) serviços prestados}

De certa forma, o grupo foi unânime em seu desconhecimento sobre os serviços oferecidos pela Biblioteca. Nesse sentido, consideram a divulgação deficitária.

Problemas específicos foram mencionados em relação ao serviço de reprografia, no que diz respeito tanto a seu custo como à demora para atendimento.

\section{PRINCIPAIS RESULTADOS E EXPEC- TATIVAS}

Tudo leva a crer que a reforma foi fundamental para a melhoria do ambiente físico. Segundo a opinião dos participantes, houve uma mudança positiva no comportamento de toda equipe da biblioteca. Da mesma forma, a partir da manifestação dos participantes, é possível acreditar que os próprios usuários da biblioteca sentiram a melhora do ambiente e passaram a preservar mais não só o acervo como também o próprio espaço físico.

Pode-se observar, confirmando a afirmativa de Hernon \& Altman (1996), que a forma de recuperação da informação utilizada pelos bibliotecários nem sempre é facilmente compreendida pelos usuários (tanto é assim, que um dos membros do grupo citou que o aspecto positivo do livre acesso às estantes é a possibilidade de encontrar obras que não se conseguiu localizar nos catálogos).

Além disso, constatou-se que a forma de utilização dos recursos de informática da biblioteca não está muito clara para os usuários. Conforme esclareceram na entrevista, sentiram como muito positiva a aquisição e ampliação das máquinas colocadas à sua disposição, mas consideram que ainda necessitam de informações para a utilização das bases on-line e do catálogo automatizado.

Como mencionado, o grupo foi unânime em seu pouco conhecimento sobre os serviços prestados pela biblioteca. Destaque-se, nesse sentido, que afirmaram desconhecer os Sumários de Periódicos, publicados pelo Serviço de Biblioteca e Documentação, um instrumento bibliográfico considerado, pelos bibliotecários, como importante fonte de pesquisa e atualização.

Quanto à qualidade no atendimento, o grupo manifestou interesse em maior autonomia na biblioteca. Nesse sentido, confirmou as observações de Hernon \& Altman (1996) que, em seu trabalho, salientam essa preocupação por parte dos usuários.

O único aspecto do serviço sobre o qual não se apontou melhora significativa diz respeito ao setor de reprografia, sobre o qual o grupo deixou clara sua insatisfação. Sob esse aspecto, a experiência com o grupo de foco foi bastante positiva para a biblioteca, pois evidenciou a permanência de problemas para os quais se pensava ter encontrado soluções satisfatórias: desde 1998, por decisão administrativa, optou-se pela 
terceirização desse serviço, esperando-se com isso atingir maior nível de eficiência e satisfação dos usuários. A discussão demonstrou que muito ainda necessita ser feito nesse sentido.

Em termos de necessidades e expectativas manifestadas pelo grupo, podem ser destacadas as seguintes:

- manter uma boa cópia das partituras para circulação, ao mesmo tempo que se realiza uma atividade de restauração e guarda dos originais, pois boa parte dos existentes encontrase rabiscada;

- revisar as fitas cassete, pois elas estão velhas e o som já não tem a mesma fidelidade original; deve-se também aumentar o número de CDs e ampliar as gravações em fitas;

- realizar um trabalho de conscientização dos usuários para que zelem pela preservação dos materiais

- colocar mais câmaras de vídeo, para inibir a depredação e furto do acervo

- providenciar novas aquisições ou assinaturas de periódicos e completar as falhas existentes;

- divulgar melhor os serviços oferecidos;

$\mathrm{Na}$ visão do grupo, o que se espera, neste momento de adaptação para um novo espaço físico é a estabilização da equipe da biblioteca. Segundo o grupo, antes não existiam condições de trabalho adequadas e não havia estimulo para 102 a utilização e permanência no espaço, o que passou a ocorrer desde a inauguração do novo ambiente.

Por outro lado, causou uma certa surpresa aos pesquisadores a constatação de que o grupo, neste momento, não possui grandes necessidades a serem satisfeitas nem tampouco cultiva muitas expectativas em relação aos serviços.

\section{CONCLUSÃO}

Apesar das dificuldades encontradas para formação do grupo e das limitações que o trabalho eventualmente pode ter apresentado, considera-se que a experiência foi de grande valor tanto para a biblioteca quanto para os participantes do grupo, permitindo o aprofundamento de questões importantes para a melhoria da qualidade dos serviços. Destaque-se, ainda, que, a atuação de alguém na qualidade de moderador e observador em um processo entrevista em que o objeto de estudo é o seu próprio ambiente de trabalho revelou-se um interessante exercício de controle pessoal. Por outro lado, foi também importante compreender que, ao final, esta dupla função possibilitou esclarecer os participantes quanto à proposta da pesquisa e, ao mesmo tempo, garantir que a presença de membros da equipe de coordenação da biblioteca na orientação dos trabalhos não gerasse qualquer tipo de inibição. Dessa forma, considera-se que os objetivos da entrevista foram atingidos.

Considerando-se os pontos acima levantados, pretende-se, na medida do possível, implementar as sugestões/informações obtidas na entrevista, sem descuidar das limitações enconInf.Inf., Londrina, v. 4, n. 2, p. 95-104, jul./dez. 1999 
tradas em função da representatividade dos participantes em relação a todos os departamentos da Escola. À exceção daquelas para aquisição de recursos de informação específicos, que não dependem exclusivamente do SBD/ECA e sim de políticas adotadas pela Universidade de São Paulo, a maioria das sugestões apresentadas são de simples aplicabilidade, não exigindo maiores investimentos.

Em função dos resultados obtidos com a utilização da técnica de entrevista por grupo de foco, decidiu-se incluir esse tipo de atividade no planejamento do SBD/ECA para os próximos exercícios, utilizando-o em paralelo com outros métodos de obtenção de opiniões. Por intermédio dele, pode-se constatar como é produtivo ouvir diretamente os usuários e permitir que estes se manifestem de forma espontânea e sem inibições, algo difícil de se obter com uma entrevista tradicional ou um questionário. Nesse sentido, o trabalho realizado apenas corroborou as informações dos autores consultados, evidenciando a viabilidade dessa técnica para o ambiente de informação acadêmica.

\section{REFERÊNCIAS BIBLIOGRÁFICAS}

FRASER, Colin, RESTREPO-ESTRADA, Sonia. Focus group discussions in development work: some field experiences and lessons learned. The Journal of Development Communication, v. 9, n. 1, p. 68-84, 1998.

DRABENSTOTT, Karen Markey. Focused group interviews. IN: GLAZIER, J. D., POWELL, R. R. Qualitative research in information management. Englewood:
Libraries Unlimited, 1992. p. 85-104

HERNON, Peter, ALTMAN, Ellen. Service quality in academic libraries. Norwood : Ablex, 1996. 187p.

MORGAN, David. L. Focus groups as qualitative research. Newbury Park: Sage, 1988. 85p.

PATTON, Michael Quinn. Qualitative evaluation and research methods. 2.ed. Newbury Park : Sage, 1990.

RADFORD, Marie L. Approach or avoidance? The role of nonverbal communication in the academic library user's decision to initiate a reference encounter. Library Trends, v. 46, n. 4, p. 669-717, 1998.

ROSE, Pamela M., STOKLOSA, Kristin, GRAY, Sharon A. A focus group approach to assessing technostress as the reference desk. Reference \& User Services Quarterly, v. 37, n. 4, p. 311-17, 1998.

ST. CLAIR, Guy. Customer service in the information environment. London : BowkerSaur, 1995. 143p.

WIDDOWS, R., HENSLER, T. A., WYNCOTT, M. H. The focus group interview: a method for assessing users'evaluation of library service. College \& Research Libraries, v. 52, n. 4, p. 352-359, 1991.

Os autores agradecem a colaboração da Sra. Elaine Pereira, assistente administrativa do SBD/ ECA, pelo auxílio na realização da entrevista. 


\section{Bárbara Júlia Leitão}

Diretora Técnica do Serviço de Biblioteca e Documentação da Escola de Comunicações e Artes (SBD/ECA) da Universidade de São Paulo. Brasil.

\section{Waldomiro Vergueiro}

Professor Doutor do Departamento de Biblioteconomia e Documentação da Escola de Comunicações e Artes da Universidade de São Paulo. Brasil.

\section{Title}

The use of focus group approach for assessing customer's views: the experience of the Library and Documentation Service of ECA/USP

\section{Abstract}

Having the international literature in information sciences as a starting point, it discusses the focus group approach and its use in the information services environment. It describes and analyses an experience with this technique in the School of Communications and Arts of the University of São Paulo, Brazil. It aims at demonstrating its viability for academic libraries in developing countries, arguing that it provides valuable data for the improvement of quality in the services. It also proposes the integration of this technique to library planning.

\section{Keywords}

Libraries - Planning - Focus group approach; Users

- Interview - Focus group approach; Focus group approach

\section{Título}

La utilización del grupo de foco para la evaluación de la opinión de los clientes: la experiencia del servicio de biblioteca y documentación de la ECA/USP

\section{Resumen}

Trata de la técnica de estrevista por medio de grupo de foco y su utilización en ambientes de servicio de información, partiendo de la literatura internacional sobre el tema. Describe y analisa experiencia realizada en la biblioteca de la "Escola de Comunicações e Artes da Universidade de São Paulo", utilizando esa técnica de investigación cualitativa. Busca demostrar su viabilidad para bibliotecas acadêmicas de países en desarrollo, defendiendo que ella propicia el alcance de resultados valiosos para la mejoría de la cualidad de los servicios ofrecidos. Propone la integración de la técnica a los procesos de planteamiento bibliotecario.

\section{Palabras-clave}

Bibliotecas - Planteamiento - Grupo de foco; Usuarios - Entrevista - Grupo de foco; Grupo de foco.

Artigo recebido em: 14/05/99 